\title{
BiblioMadSalud, cooperando en un espacio común
}

\author{
Comité Ejecutivo de BiblioMadSalud: Elena Primo-Peña ${ }^{1}$, Concepción Campos-Asensio ${ }^{2}$, María- \\ Isabel Domínguez-Aroca ${ }^{3,}{ }^{*}$, Oliver Martín Martín ${ }^{4}$, Isabel Martínez-Hervás ${ }^{5}$, Juan Medino- \\ Muñoz ${ }^{6}$, María José Rebollo Rodríguez ${ }^{7}$, José Carlos Redondo García ${ }^{8}$, José-Manuel Estrada- \\ Lorenzo $^{9}$ \\ 1 Biblioteca Nacional de Ciencias de la Salud, Instituto de Salud Carlos III \\ 2 Biblioteca, Hospital Universitario de Getafe \\ Biblioteca, Universidad de Alcalá \\ Biblioteca, Universidad Complutense de Madrid \\ Biblioteca, Hospital Universitario Severo Ochoa \\ Biblioteca, Hospital Universitario de Fuenlabrada \\ Biblioteca, Ilustre Colegio Oficial de Médicos de Madrid \\ Biblioteca Virtual de la Consejería de Sanidad de la Comunidad de Madrid \\ Biblioteca, Hospital Universitario 12 de Octubre \\ * Autor correspondencia: misabel.dominguez@uah.es;
}

Recibido: 29/04/2019; Aceptado: 15/05/2019; Publicado: 31/05/2019

Resumen: Introducción: BiblioMadSalud (BMS) surge en 2015, como una red informal de profesionales de bibliotecas en activo que tienen como denominador común la temática (ciencias de la salud) y la ubicación geográfica (Comunidad de Madrid) pero pertenecientes a distintas administraciones (estatal y autonómica), contextos (Administración, hospitales, organizaciones profesionales, universidades y organismos públicos de investigación) y ámbitos (público y privado) con el objetivo de promover la coordinación y colaboración entre sus instituciones, en un formato organizativo innovador a nivel regional y nacional. Material y método: análisis descriptivo del proceso y desarrollo de BiblioMadSalud. Resultados: BibliomadSalud se ha desarrollado a partir de sucesivas etapas: 1 . Génesis: diseño y planificación de su identidad, funcionalidades y estructura; 2. Organización: creación de sus órganos competentes como Comité Ejecutivo, Grupos de Trabajo y Asamblea; e. Puesta en marcha: elaboración de un directorio geolocalizado de bibliotecas, organización de tres jornadas de participación asamblearia, constitución de 10 grupos de trabajo (BiblioBaremo, BiblioMOOCsalud, Estadísticas, Contribuyendo al OA, Callejeando, Recursos de aprendizaje, \#BiblioRRHH, BiblioEvalúa, BiblioEstructura y BiblioVisibilidad), organización de actividades formativas trimestrales y difusión en redes sociales. Conclusiones: la colaboración entre instituciones de distintas administraciones, contextos y ámbitos, siempre resulta enriquecedora; trabajar organizados en Grupos de Trabajo con objetivos concretos facilita la visibilización de resultados; las actividades formativas periódicas han contribuido a una mayor cohesión del colectivo; la constitución como red informal, aunque útil en el momento de arranque, dará paso a alguna estructura asociativa más estable para la continuidad de BMS.

Palabras Clave: Cooperación bibliotecaria; Bibliotecarios de ciencias de la salud; Madrid; Asociaciones profesionales.

Abstract: Introduction: BiblioMadSalud (BMS) emerges in 2015 as an informal network for library professsionals in active service that have as common denominator their discipline (Health Sciences) and geographical location (Comunidad de Madrid), bur separated by their dependence of different government bodies (Central State or Autonomous Communities), contexts (Administration, Hospitals, Professional Organizations, Universities and Public Research Organizations) or sector (publico $\mathrm{r}$ private). Its goa lis to promote coordination and collaboration between their instituions through an innovative organizational model that Works at regional an national level. Methods: Descriptive análisis of the genesis and development of BiblioMadSalud. Results: BiblioMadSalud 
has evolved over successive stages: 1: Genesis: design and planning of its identity, functionalities and structure; 2.Organization: creation of thir competent bodies, such as the Executive Committee, task groups or Assembly; 3. Impelentation: creation of a geolocated directory of libraries, organization of three anual meeting of participation in the Assemby, formation of 10 task groups (BiblioBaremo, BiblioMOOCsalud, Estadísticas, Contribuyendo al OA, Callejeando, Recursos de aprendiaaje, \#BiblioRRHH, BiblioEvalúa, BiblioEstructura y BiblioVisibilidad), organization of quarterly education activities and dissemination on social networks. Conclusion: cooperation betwwwn institutions from different administrations, contexts and sectors is always rewarding; working in task groups with particular objetives helps to make the results visible; periodic educational activities contribute to a better cohesiono $f$ the collective; the constitution as an informal network, though useful at firt, Will give way to a more stable associative structure for the continuity of BMS.

Key words: Librarian cooperation; Health librarians; Madrid; Professional associations.

\section{Introducción}

En el ámbito de las bibliotecas, la cooperación entre sus profesionales es una tradición, una necesidad y un hábito, pero la cooperación institucional no ha sido siempre tan fluida debido sobre todo a los ámbitos organizativos en que éstas se desenvuelven (estatal, autonómico y universitario). La red de bibliotecas de salud a nivel estatal [1] ha generado una cooperación periódica, por ejemplo, en lo que al servicio de obtención de documentos se refiere o a la organización periódica de las Jornadas Nacionales, pero adolece de un sistema permanente de cooperación.

Por ello, BiblioMadSalud surge en 2015 como una red informal de profesionales en activo que tienen como denominador común su ámbito temático (las ciencias de la salud) y su ubicación geográfica (la Comunidad de Madrid), con el aliciente de pertenecer a distintas administraciones (estatal y autonómica), contextos (administración, hospitales, organizaciones profesionales, universidades y organismos de investigación) y ámbitos (público y privado) con el objetivo de promover la cooperación y la coordinación entre sus instituciones y sus profesionales, en un formato organizativo innovador a nivel regional y nacional por su carácter integrador e informal [2].

Tres son los principios fundamentales que sustentan su existencia y actividades:

1. Cohesión (integrar componentes dispares por su procedencia pero similares en sus objetivos y actividades, a través de actividades formativas comunes, grupos de trabajo y reuniones periódicas).

2. Visibilidad (dar difusión a las actividades, resultados y productos de los grupos, del colectivo y de las bibliotecas individuales, a través de todos los medios disponibles (blog, redes sociales, jornadas, congresos, actividades formativas, etc.) con el propósito de hacer visible una actividad profesional que resulta invisible para buena parte de la sociedad, manifestando así la importancia de las bibliotecas de salud en el ámbito sanitario (en sus tres facetas principales: asistencia, formación e investigación).

3. Participación (de sus miembros en el desarrollo de BiblioMadSalud y en el desarrollo de sus bibliotecas principalmente a través de su asamblea anual y sus reuniones, pero también mediante grupos y actividades abiertos a las ideas de cualquiera de sus miembros).

Por su definición, BiblioMadSalud es un grupo heterogéneo (Figura 1), posiblemente más que otras agrupaciones geográficas autonómicas, dado que, por ejemplo en la ciudad de Madrid confluyen tres administraciones: municipal, autonómica y estatal [3]. 


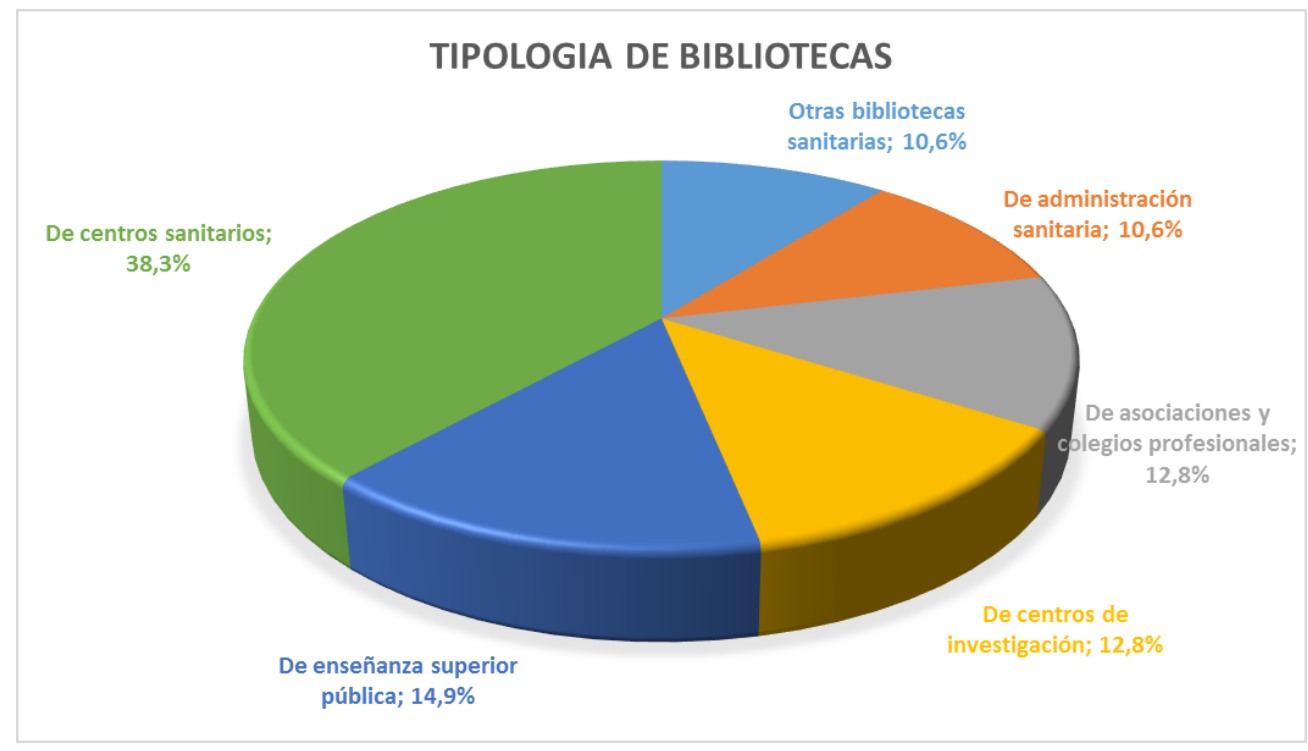

Figura 1 Tipología de las bibliotecas de ciencias de la salud de la Comunidad de Madrid.

Esta heterogeneidad es precisamente su mayor riqueza y valor, pero su variedad implica realizar un esfuerzo para construir lazos e intereses comunes donde cada miembro dispar y distinto aporte su personalidad diferenciadora. De ahí su fortaleza, la integración cohesionada de elementos diferentes que se enriquecen en el intercambio y la complementariedad, abandonando posturas inmovilistas e individuales en beneficio del grupo.

Esta mencionada cohesión no puede construirse de la nada sino dotando al grupo de herramientas que activen esos nexos comunes, como pueden ser la organización de pequeños grupos de trabajo (que con sus conclusiones y resultados ayuden a crecer al colectivo), actividades formativas trimestrales (que ayuden a aprender de forma continuada), jornadas anuales (que faciliten la discusión y el desarrollo organizativo) y la creación de recursos comunes (catálogos, bases de datos, webs, cursos, documentos de posicionamiento ante temas que afectan al colectivo, ...) que a la vez fomentan la visibilidad, alimentan la idea de pertenencia a un grupo activo.

La visibilidad de BiblioMadSalud nace también del trabajo constante del Comité Ejecutivo, de sus grupos de trabajo y de sus miembros. Un trabajo que se materializa en la Jornada anual (en 2019 se celebrará la tercera) de carácter asambleario (donde no sólo se aprende con la participación de ponentes externos sino que se discute y se toman decisiones organizativas que afectan al colectivo), en la existencia de una página web (Figura 2) que trasluce todas las actividades generales y la evolución de los grupos, la presencia en otras Jornadas y Congresos, la elaboración de recursos comunes (entre los que figura ya un directorio y otros productos en proceso de elaboración, como por ejemplo, un manual de buenas prácticas de las bibliotecas de ciencias de la salud), las conclusiones de los grupos de trabajo (por ejemplo, una propuesta de baremos para procesos de selección y otra de perfil profesional bibliotecario) y la proyección al exterior a través de los eventos formativos y su participación en redes sociales. 

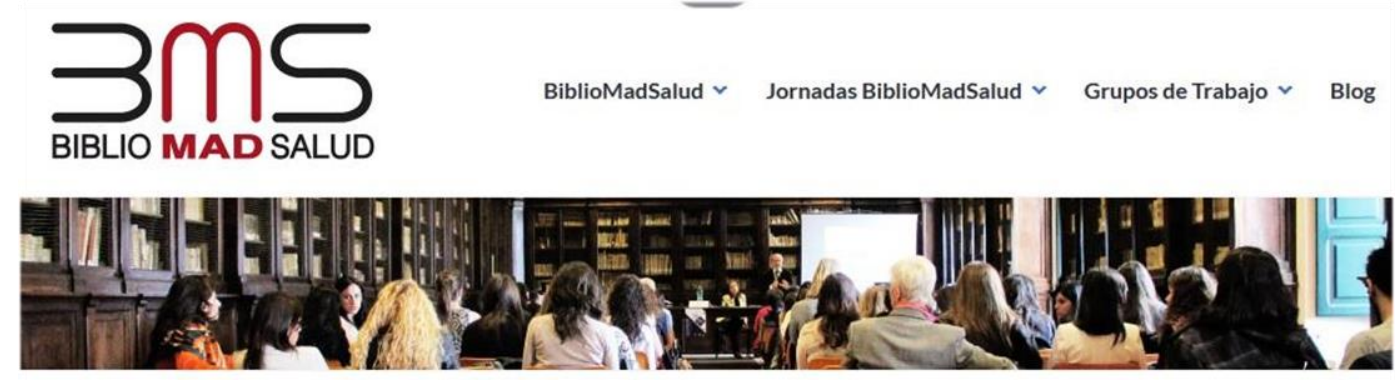

\section{BiblioMadSalud}

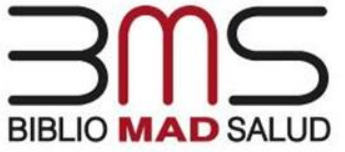

participantes. es el colectivo de profesionales de las bibliotecas de Ciencias de la Salud en la Comunidad de Madrid. El objetivo de esta iniciativa es promover la coordinación y colaboración entre las instituciones

El 29 de noviembre de 2016 se celebró la I Jornada BiblioMadSalud 2016 en el Colegio de Médicos de Madrid con un claro objetivo fundacional y en la esperanza de que a partir de ese primer encuentro surgieran iniciativas interesantes y productivas para el colectivo.

Figura 2 Web de BiblioMadSalud

La estructura organizativa de BiblioMadSalud consta de los siguientes órganos:

- $\quad$ Comité Ejecutivo

- Asamblea general (Figura 3)

- Comité organizador de Jornada anual

- Grupos de trabajo

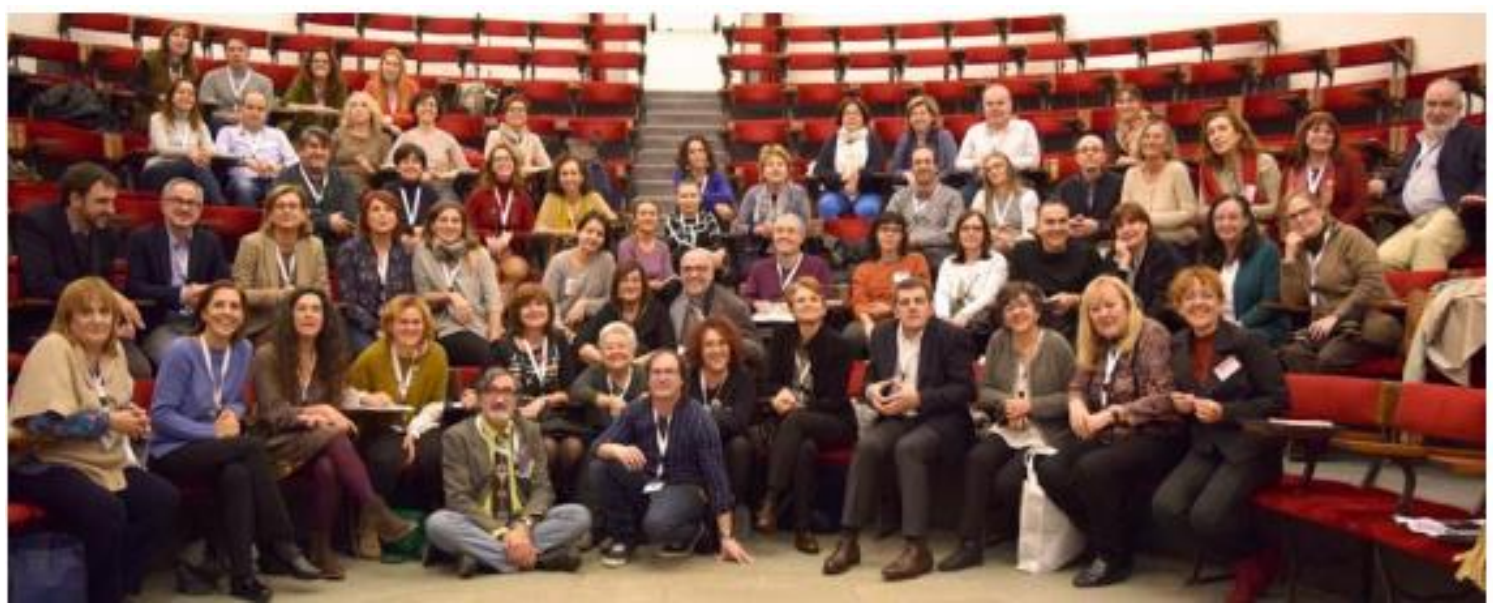

Figura 3 I Jornada BiblioMadSalud 
El Comité Ejecutivo tiene una vigencia de 2 años y está constituido por un equipo de entre 5-10 profesionales del sector, que han sido elegidos por la asamblea previa presentación de candidatura y cuenta con representación de los distintos tipos de bibliotecas (administración, organizaciones profesionales, universitarias, hospitalarias y científicas). Son sus funciones:

- Coordinación general del colectivo y representatividad entre jornadas (reuniones trimestrales).

- Gestión de la comunicación a través de la página web (alojamiento de documentos, información de las jornadas y actividades programadas, mantenimiento del dominio y apoyo al Comité Organizador de las Jornadas) [https://bibliomadsalud.wordpress.com/], del correo electrónico [bibliomadsalud@icomem.es] y de las redes sociales mediante Twitter [@BiblioMadSalud], canal de YouTube [https://www.youtube.com/channel/UCdRJc1BPaKaBgGPAmBYoHrQ] y el blog [https://bibliomadsalud.wordpress.com/blog/]

- $\quad$ Actualización del directorio de bibliotecas de ciencias de la salud en Madrid:

GeoBiblioMadSalud (Figura4)

[https://www.google.com/maps/d/u/1/edit?mid=1yoYoub tL0Gb9JYgMWety2ZMdAw\&ll=40. 23044937608054\%2C-3.743796533398381\&z=11].

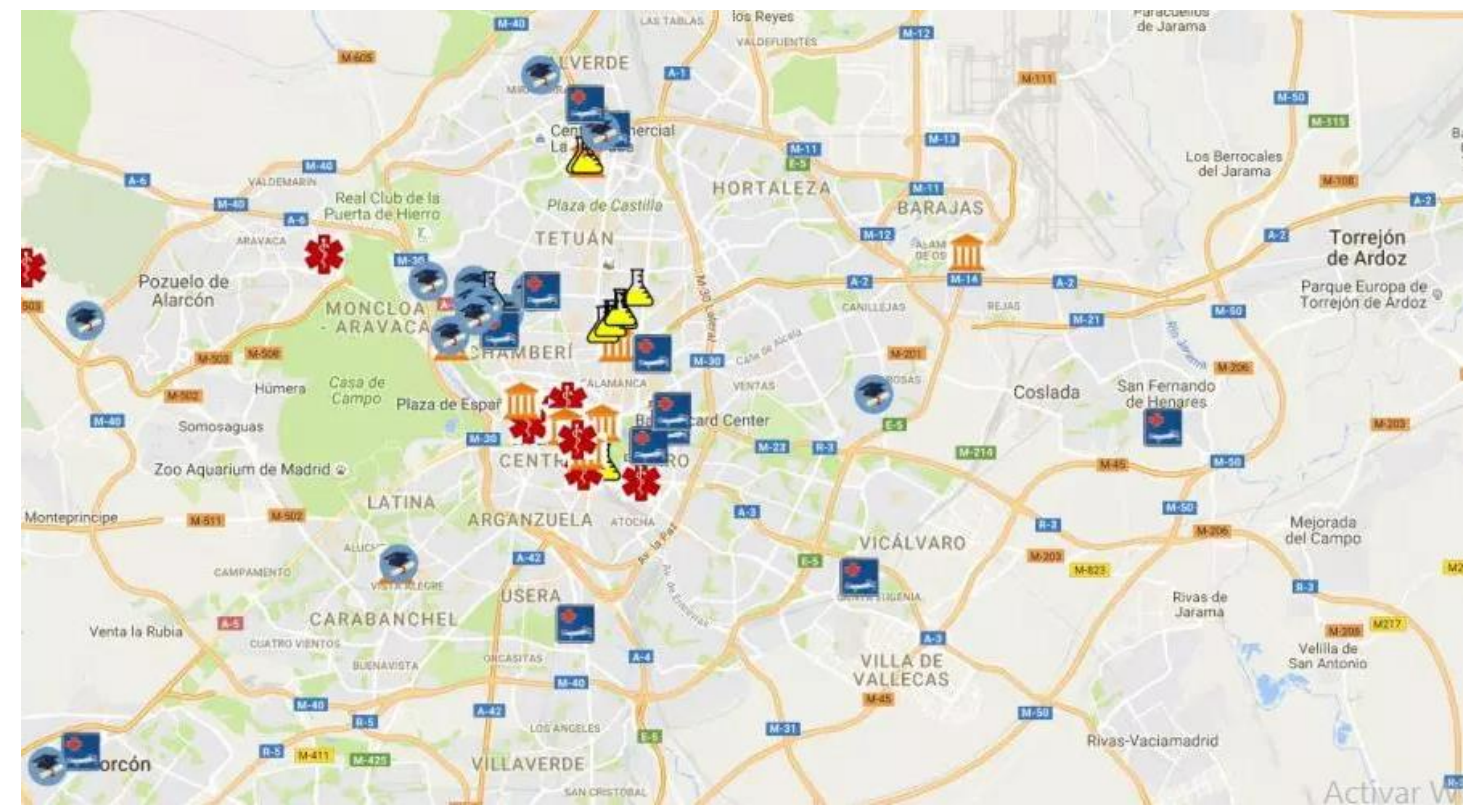

Figura 4. GeoBiblioMadSalud

- Apoyo al Comité organizador de la Jornada anual, a cuyas reuniones acudirán siempre al menos dos de sus miembros; $y$

- Organización de otras convocatorias (acciones formativas, encuentros, etc.) que se pudieran plantear entre Jornadas BiblioMadSalud. 
La Asamblea general está compuesta por profesionales en activo de bibliotecas de ciencias de la salud en Madrid y se reúne al menos una vez al año en una sesión de trabajo (Jornada). Supervisa la actividad del Comité Ejecutivo, aprueba y/o sanciona las líneas de trabajo a acometer, códigos y demás documentos que emanen de la organización y vota entre las distintas candidaturas de Comité Ejecutivo que se presenten. Ha celebrado tres reuniones anuales desde 2016 (Tabla I):

Tabla I Jornadas BiblioMadSalud

\begin{tabular}{|c|c|c|}
\hline Nombre & Fecha & Asistentes \\
\hline I Jornada BMS & $28 / 11 / 2016$ & 62 \\
\hline I Reunión BMS & $07 / 06 / 2017$ & 50 \\
\hline II Jornada BMS & $21 / 06 / 2018$ & 93 \\
\hline III Jornada BMS & $23-24 / 05 / 2019$ & \\
\hline
\end{tabular}

El Comité Organizados de la Jornada Anual tiene vigencia de 1 año y es el encargado de la organización de la Jornada anual de trabajo. Se crea a partir de la candidatura que obtenga la confianza de la Asamblea general o a instancias del Comité Ejecutivo si ninguna institución se postulase para ello. No tiene un número máximo de integrantes pero deber reservar dos puestos para miembros del Comité Ejecutivo para una buena coordinación entre ambos comités. Puede delegar los cometidos de diseño del programa y valoración de comunicaciones en un comité científico creada para la ocasión.

Los Grupos de Trabajo son creados a instancias del Comité Ejecutivo para concretar líneas de trabajo de BiblioMadSalud o propuestos/presentados por cualquier miembro del colectivo como proyectos en la reunión anual de la Asamblea. Su vigencia suele ser anual, aunque pueden ser prolongados si su actividad lo requiere. Los grupos informarán a la Asamblea general de los resultados obtenidos durante el año y contarán con el apoyo y seguimiento del Comité Ejecutivo. Desde los inicios de BiblioMadSalud se han constituido diez grupos (de los que siguen vigente ocho):

- BiblioBaremo (2017-2018) [4]: su objetivo ha sido ofrecer un documento profesional, elaborado por profesionales de las bibliotecas, que sirva como estándar a políticos y gestores en las convocatorias de profesionales sanitarios en el apartado de méritos bibliográficos / investigación.

- BiblioEstructura 82018- ): cuyos objetivos son trabajar por el reconocimiento de las bibliotecas especializadas en ciencias de la salud, en el reglamento de su estructura, organización y funcionamiento de su institución, y proponer un protocolo de funcionamiento de dichas bibliotecas.

- BiblioEvalúa (2018- ): se ha propuesto servir de apoyo a los bibliotecarios en el asesoramiento a investigadores e instituciones para aumentar la visibilidad de su producción científica y mejorar su impacto, elaborando para ello una guía.

- BiblioMOOCSalud (2017- ) [5]: su objetivo es crear NOOCs o MOOCs para la formación y puesta al día de los profesionales de las bibliotecas de ciencias de la salud.

- \#BiblioRRHH (2017- ): interesado en definir funciones y tareas del colectivo bibliotecario de ciencias de la salud en Madrid para la elaboración de un perfil profesional específico.

- BiblioVisibilidad (2018- ): se propone diseñar una guía para el desarrollo de un plan de marketing para las bibliotecas de ciencias de la salud, establecer un marco de colaboración con EAHIL (European Association for Health Information and Libraries) y promocionar estancias de intercambio de corta duración, entre bibliotecas de ciencias de la salud dentro del marco del programa HOPE (Hospitals for Europe) o cualquier otro vigente. 
- Callejeando (2016- ): ha desarrollado un mapa de las ciudades de Madrid, Fuenlabrada, Leganés y Majadahonda, rastreando la información relativa a médicos y otros profesionales de la salud en calles, edificios y monumentos.

- Contribuyendo al OA (2016- ): su objetivo es formar a los profesionales de las bibliotecas de salud en el acceso abierto y promover así la formación de los usuarios.

- Estadísticas (2016- ): se encarga de recabar datos actuales de las bibliotecas de salud (personal, recursos, gastos,...).

- Recursos de aprendizaje (2016- en suspenso): se propone modificar sus objetivos para lograr su viabilidad.

\section{BiblioMadSalud se ha desarrollado desde 2015 en sucesivas etapas:}

1. Génesis: donde se diseñó y planificó su identidad, a partir de la presentación en su Primera Jornada en 2016 de diferentes modelos de agrupación (formal, informal y mixta), una vez identificada la necesidad y utilidad, dada la proximidad geográfica, de una mayor y constante contacto profesional entre unas bibliotecas y unos profesionales que compartían un especio común y unas similares preocupaciones.

2. Organización: un Comité provisional, que organizó la Primera Jornada se encargó de diseñar un embrión de Agrupación donde sus órganos competentes permitieran una organización flexible y una toma de decisiones ágil, a la vez que se diera cabida a todas las sensibilidades y tipologías, por ello se creó un Comité Ejecutivo como cabeza visible, unos grupos de trabajo como actores ejecutantes de procesos y productos y una Asamblea para la toma de decisiones colectiva.

3. Puesta en marcha: desde la segunda Jornada ya tomaron cuerpo los distintos órganos del colectivo y comenzaron a vislumbrar los primeros resultados cooperativos a partir de los cursos de formación y los primeros resultados de los grupos de trabajo (BiblioBaremos y Contribuyendo al OA).

\section{Resultados}

El resultado más evidente, tras casi cuatro años de trabajo, es la existencia del colectivo BiblioMadSalud, que engloba 89 bibliotecas de salud, que realiza actividades formativas trimestrales, que ha congregado a cerca de cien profesionales en cada una de sus sesiones anuales (profesionales que no se reunían hasta entonces con esa periodicidad), que ha integrado intereses de profesionales diferentes (universidades, hospitales, administración colegios y hospitales), con un objetivo común (trabajar en beneficio de las biblioteca, sus usuarios y sus profesionales), que ha generado sinergias que han facilitado el trabajo en grupo de más de 40 profesionales), que ha promovido la convivencia de usuarios-profesionales y proveedores en un mismo entorno de discusión y aprendizaje, y lo más importante, que si no se hubiera constituido todos estos resultados a día de hoy no existirían.

Ejemplos concretos de la actividad de los grupos de trabajo (Tabla II) serían:

- Un curso de formación de formadores sobre Acceso Abierto (GT Contribuyendo al OA).

- Un documento elaborado por bibliotecarios/as que podría servir como estándar a gestores en convocatorias de profesionales sanitarios para el apartado de méritos bibliográficos y de investigación (GT BiblioBaremo).

- Un diseño de perfil profesional del bibliotecario/documentalista especializado en ciencias de la salud, funciones, habilidades y competencias (GT \#BiblioRRHH).

- Un curso MOOC en 2018, en colaboración con la UNED, sobre "Identidad digital, posicionamiento y promoción de los profesionales de ciencias de la salud" en el que han 
colaborado distintos perfiles profesionales e instituciones (bibliotecarios, profesores universitarios, profesionales sanitarios, periodistas especializados) y que han contado con más 672 alumnos, de los cuales 127 solicitaron acreditación y 545 fueron oyentes (GT BiblioMOOCSalud).

Tabla II Grupos de Trabajo

\begin{tabular}{|c|c|c|c|}
\hline $\begin{array}{c}\text { Denominación del } \\
\text { Grupo }\end{array}$ & № de integrantes & $\begin{array}{l}\text { Año de puesta } \\
\text { en marcha }\end{array}$ & Resultados \\
\hline BiblioBaremo & $8+2$ asesores & 2017 & $\begin{array}{l}\text { Documento de estándares. } \\
\text { Publicación en la revista } \\
\text { fisioGlia - enero 2019. Difusión } \\
\text { en reunión anual Bibliosaùde } \\
2018\end{array}$ \\
\hline BiblioEstructura & $3+1$ asesor & 2018 & En proceso \\
\hline BiblioEvalúa & $5+1$ asesor & 2018 & En proceso \\
\hline BiblioMOOCSalud & $\begin{array}{c}6+20 \\
\text { colaboradores }\end{array}$ & 2017 & $\begin{array}{l}\text { MOOC sobre "Identidad digital, } \\
\text { posicionamiento y promoción de } \\
\text { los profesionales de ciencias de } \\
\text { la salud". Plataforma UNED } \\
\text { Abierta. } 24 \text { vídeos en YouTube. }\end{array}$ \\
\hline \#BiblioRRHH & $9+2$ asesores & 2017 & En proceso \\
\hline BiblioVisibilidad & 6 & 2018 & En proceso \\
\hline Callejeando & $9+2$ asesores & 2016 & $\begin{array}{l}\text { Presentado el mapa en Leganés } \\
\text { en } 2017 \text { en el Hospital } \\
\text { Universitario Severo Ochoa }\end{array}$ \\
\hline $\begin{array}{l}\text { Contribuyendo al } \\
\text { OA }\end{array}$ & $2+2$ asesores & 2016 & $\begin{array}{l}\text { Curso de formación de } \\
\text { formadores en Acceso Abierto }\end{array}$ \\
\hline Estadísticas & 4 & 2016 & $\begin{array}{l}\text { Con datos estadísticos de los } \\
\text { años } 2015 \text { y } 2017 \text { ( } 32 \\
\text { indicadores) }\end{array}$ \\
\hline
\end{tabular}

Otro de los elementos de cohesión interna del colectivo, además de motor de visibilidad y apertura hacia los profesionales del entorno han sido las actividades formativas (seminarios, cursos y jornadas breves) (Tabla III):

Tabla III. Actividades formativas

\begin{tabular}{|l|c|c|}
\hline \multicolumn{1}{|c|}{ Nombre } & Fecha & Asistentes \\
\hline Formación en acceso abierto & $14-28 / 11 / 217$ & 30 \\
\hline OVID Day & $13 / 12 / 2017$ & 193 \\
\hline Seminario Bibliometría & $11-12 / 04 / 2018$ & 75 \\
\hline EBSO Lunch \& Learn & $18 / 05 / 2018$ & 95 \\
\hline Comunicación eficiente con gráficos & $22-23 / 11 / 2018$ & 50 \\
\hline Jornada Cochrane & $13 / 03 / 2019$ & 80 \\
\hline
\end{tabular}

Para la difusión de las informaciones, eventos, formaciones, informes y resultados nos hemos apoyado sobre todo en herramientas de las redes sociales, y en las jornadas y actividades formativas, hemos utilizado hasthgs para unificar la consulta y visibilidad (\#BMS2016, \#BMS18, \#BMSOVIDAY, ...) en beneficio de la cohesión y visibilidad del colectivo. 
Queda mucho camino por recorrer en busca de esa cohesión, visibilidad y participación planteadas como principios del colectivo, y entre sus líneas de trabajo futuras figuran su constitución como asociación para dar carta de naturaleza a su existencia como grupo informal de bibliotecarios, la creación de recursos comunes de trabajo que optimicen las tareas individuales y una mayor cooperación entre usuarios y profesionales que trascienda las actividades formativas, único ámbito en el que hasta ahora se ha materializado la coexistencia ente usuarios y profesionales.

\section{Conclusiones}

La colaboración entre instituciones de distintas administraciones, contextos y ámbitos, siempre resulta enriquecedora, trasciende la mera cooperación profesional individual, que es una tradición en las bibliotecas de salud, y se institucionaliza, lo que facilita el uso en común de recursos profesionales y tiempos de trabajo.

Trabajar organizados en grupos de trabajo con objetivos concretos facilita la visibilización de resultados y contribuye a la interacción y trabajo en equipo de los profesionales.

Las actividades formativas periódicas han contribuido a una mayor cohesión del colectivo, y han permitido la convivencia, en un mismo espacio y tiempo, de bibliotecarios, profesionales sanitarios y editores, los tres elementos básicos del ámbito de la información y documentación en salud.

La constitución como red informal, aunque útil en el momento de arranque, debería dar paso a alguna estructura asociativa más estable para la consolidación de BiblioMadSalud como grupo preocupado por el desarrollo, mejora y evolución de sus bibliotecas y sus servicios.

Agradecimientos: A todas las bibliotecas y bibliotecarios del ámbito de la salud de la Comunidad de Madrid que han participado activamente en los grupos de trabajo, las jornadas y las actividades de formación organizadas por BiblioMadSalud, por su apoyo e interés, y muy especialmente a los asesores/ expertos / colaboradores que han prestado su conocimiento y tiempo de manera desinteresa en los diferentes grupos de trabajo (en especial a Marisa, Miguel Angel, Isidro, Carlos y Alicia).

Conflictos de Intereses: los autores manifiestan que este texto con pequeñas matizaciones / actualizaciones es la comunicación presentada en las XVI Jornadas Nacionales de Información y Documentación en Ciencias de la Salud. Oviedo, 4 - 5 de abril de 2019.

\section{Referencias Bibliográficas}

1. Roqué Castella P, Beceiro López G, Tomé MJ. En busca de un modelo de red bibliotecaria: estudio comparativo de las redes de bibliotecas sanitarias y otros modelos de red. 2011. En: Bibliosalud 2011: XIV Jornadas Nacionales de Información y Documentación en Ciencias de la Salud: Sevilla: Junta de Andalucía, 77.

2. San José Montano B, Junta directiva SEDIC. Las asociaciones profesionales de bibliotecas y documentación como comunidades de valor. Bol Asoc And Bibl. 2017;32(114):195-2013.

3. Martinez-Hervás I, Martín O, Estrada JM, Redondo JC. [Grupo de trabajo de Estadísticas de BiblioMadSalud]. Presentación de los resultados de la Encuesta BiblioMadSalud 2017 [Internet]. Madrid: BiblioMadSalud; 2018 [consultado el 4 de marzo de 2019]. Disponible en: https://bibliomadsalud.files.wordpress.com/2019/01/Estadistica_BMS_2017.pdf

4. Medino Muñoz, JA, Estrada Lorenzo JM, Reillo O, Gil Carballo C, Alonso Martín ML. [Grupo de trabajo BiblioBaremo de BiblioMadSalud]. BiblioBaremo: propuesta de mejora en la evaluación del currículum vitae científico / investigador del profesional sanitario. fisioGlía. 2019;6(1):5-8.

5. Grupo de Trabajo BiblioMOOCSalud de BiblioMadSalud. Identidad digital, posicionamiento y promoción de los profesionales de ciencias de la salud [Internet]. Madrid: UNED, BiblioMadSalud, 2018 [consultado el 4 de marzo de 2019]. Disponible en: https://iedra.uned.es/courses/coursev1:UNED+IdDigSalud_001+2018/about 